Article

\title{
Investigating Public Facility Characteristics from a Spatial Interaction Perspective: A Case Study of Beijing Hospitals Using Taxi Data
}

\author{
Xiaoqing Kong ${ }^{1}$, Yu Liu ${ }^{2}$, Yuxia Wang ${ }^{2}$, Daoqin Tong ${ }^{3}$ and Jing Zhang ${ }^{1, *}$ \\ 1 College of Resources Environment and Tourism, Capital Normal University, Beijing 100048, China; \\ xiaoqing_cnu@126.com \\ 2 Institute of Remote Sensing and Geographical Information Systems, Peking University, Beijing 100871, \\ China; liuyu@urban.pku.edu.cn (Y.L.); yuxia_wang@pku.edu.cn (Y.W.) \\ 3 School of Geography and Development, The University of Arizona, Tucson, AZ 85721, USA; \\ daoqin@email.arizona.edu \\ * Correspondence: zhangjings@mail.cnu.edu.cn; Tel.: +86-10-6890-2573
}

Academic Editor: Wolfgang Kainz

Received: 27 October 2016; Accepted: 29 January 2017; Published: 6 February 2017

\begin{abstract}
Services provided by public facilities are essential to people's lives and are closely associated with human mobility. Traditionally, public facility access characteristics, such as accessibility, equity issues and service areas, are investigated mainly based on static data (census data, travel surveys and particular records, such as medical records). Currently, the advent of big data offers an unprecedented opportunity to obtain large-scale human mobility data, which can be used to study the characteristics of public facilities from the spatial interaction perspective. Intuitively, spatial interaction characteristics and service areas of different types and sizes of public facilities are different, but how different remains an open question, so we, in turn, examine this question. Based on spatial interaction, we classify public facilities and explore the differences in facilities. In the research, based on spatial interaction extracted from taxi data, we introduce an unsupervised classification method to classify 78 hospitals in 6 districts of Beijing, and the results better reflect the type of hospital. The findings are of great significance for optimizing the spatial configuration of medical facilities or other types of public facilities, allocating public resources reasonably and relieving traffic pressure.
\end{abstract}

Keywords: spatial interaction; hospital service area; public facility characteristics; classification; taxi data; Beijing

\section{Introduction}

In recent years, research on the spatial configuration of public facilities has gradually gained attention in the fields of geographic information science and urban planning. To help city planners to evaluate and analyze the effectiveness of public facility configuration, scholars have conducted many studies on the accessibility of public facilities [1-4], service areas [5-8], and equity issues [9-11]. From a spatial perspective, the basic idea of these studies is to estimate the connections between demand locations and supply locations. Traditional approaches rely mainly upon census data, travel surveys, and particular records, such as medical records.

The connection between supply and demand represents a type of spatial interaction. In geographical studies, spatial interaction refers to the link between the two places and can usually be quantified using the flows of people, goods, and capital, among others. With the support of big geo-data, we collect a massive volume of data on human movement and flow to quantify spatial interactions between places [12]. Numerous studies have been conducted to examine urban or regional structures using 
spatial interaction information extracted from social media data [13], phone call records [14], public transportation card records [15], and taxi trajectories [16]. In these studies, community detection methods, which are borrowed from network sciences, were widely adopted to delineate a study area into meaningful sub-regions according to interaction strengths. Hence, these methods can be viewed as a top-down approach. However, from a bottom-up perspective, the spatial interactions associated with a locality represent its second-order properties. We can classify a set of places based on their spatial interactions. Following this idea, Liu et al. [16] incorporated spatial interaction patterns into land use classification using an unsupervised method with taxi data from Shanghai and achieved better results.

In this study, we argue that public facilities inside a city are critical to providing essential services and therefore are closely related to the city residents. Spatial interaction between service facilities and residents should reflect the characteristics of public facilities to some extent. In fact, it has been shown that spatial interaction strengths between a hospital and its patients are dependent on the attributes of the hospital [8]. Inversely, we can determine the hospitals' properties based on their spatial interactions. Using a taxi dataset of over 17,000 taxis for three months in Beijing, we introduced an unsupervised classification method to classify 78 hospitals of six districts in Beijing from the spatial interaction perspective. Hospital service areas were then delineated based on the taxi pick-ups and drop-offs in a 200-m buffer area of the hospital, and visual analysis was conducted. Finally, we obtained spatial interaction patterns between patients and hospitals and fit them with exponent law, revealing the underlying distance effect. The results show that the patient distributions of all hospitals show the exponential law distance decay effect. The exponents, however, vary from class to class and indicate the impact of distance and hospital types. For example, compared with specialty hospitals and small municipal general hospitals, military hospitals and university hospitals are less sensitive to distance.

With the support of big data, this research links the facility properties with the spatial distributions of their customers from a spatial interaction perspective. Regarding Beijing hospitals, this study will be particularly useful because the vast majority of hospitals in the city are located in the city center. People in suburban areas flock to the city center to seek medical services, increasing the tremendous burden on the city's transportation system, particularly during the morning and evening peak periods. Therefore, understanding the patient distributions of different types of hospitals is valuable for policy makers and urban planners to optimize the spatial distribution of hospitals to ease traffic jams while maintaining service accessibility. The rest of this article is organized as follows. Section 2 introduces the data and processing. Section 3 describes hospitals' service areas and patient distribution patterns. Section 4 discusses methods and classification results and analyzes the patient distribution characteristics of different types of hospitals. Section 5 provides a discussion and conclusions.

\section{Data Description and Processing}

\subsection{Taxi Trip Data}

This research used GPS data from more than 17,000 taxis for three consecutive months (May to July 2013). The dataset contains seven fields, namely, the vehicle number, positioning time of occurrence, latitude, longitude, direction, speed and status. Before data analysis, we preprocessed the taxi trajectory data first, excluding erroneous data and deleting duplicate or incomplete records. Based on these data, we then extracted locations where the passengers are picked up or dropped off. Each trip is simplified into a vector from $\left(x_{0}, y_{0}, t_{0}\right)$ to $\left(x_{d}, y_{d}, t_{d}\right)$, where $(x, y)$ represents the location of the pick-up point (PUP) and drop-off point (DOP), and $t$ is the time of pick-up and drop-off action. These extracted trips are able to collectively represent the spatial interaction between places.

\subsection{Study Area and Hospitals}

As the second biggest city in China, Beijing has comprised 16 districts since 2015 (see also Figure 1). The six districts of Xicheng, Dongcheng, Fengtai, Chaoyang, Haidian, and Shijingshan form the core 
urban area, accounting for $8 \%$ of the total area and hosting $60 \%$ of the population and $70 \%$ of the hospitals in Beijing [17]. According to the distribution of important hospitals and taxi trip data, we selected a $24 \mathrm{~km} \times 30 \mathrm{~km}$ rectangle area covering the major urban areas of Beijing as the study area (Figure 1). To measure the spatial interaction strengths, the study area was discretized into $7201 \mathrm{~km} \times 1 \mathrm{~km}$ square cells. All trips extracted from the taxi trajectory data were aggregated based on the $1 \mathrm{~km} \times 1 \mathrm{~km}$ cells.

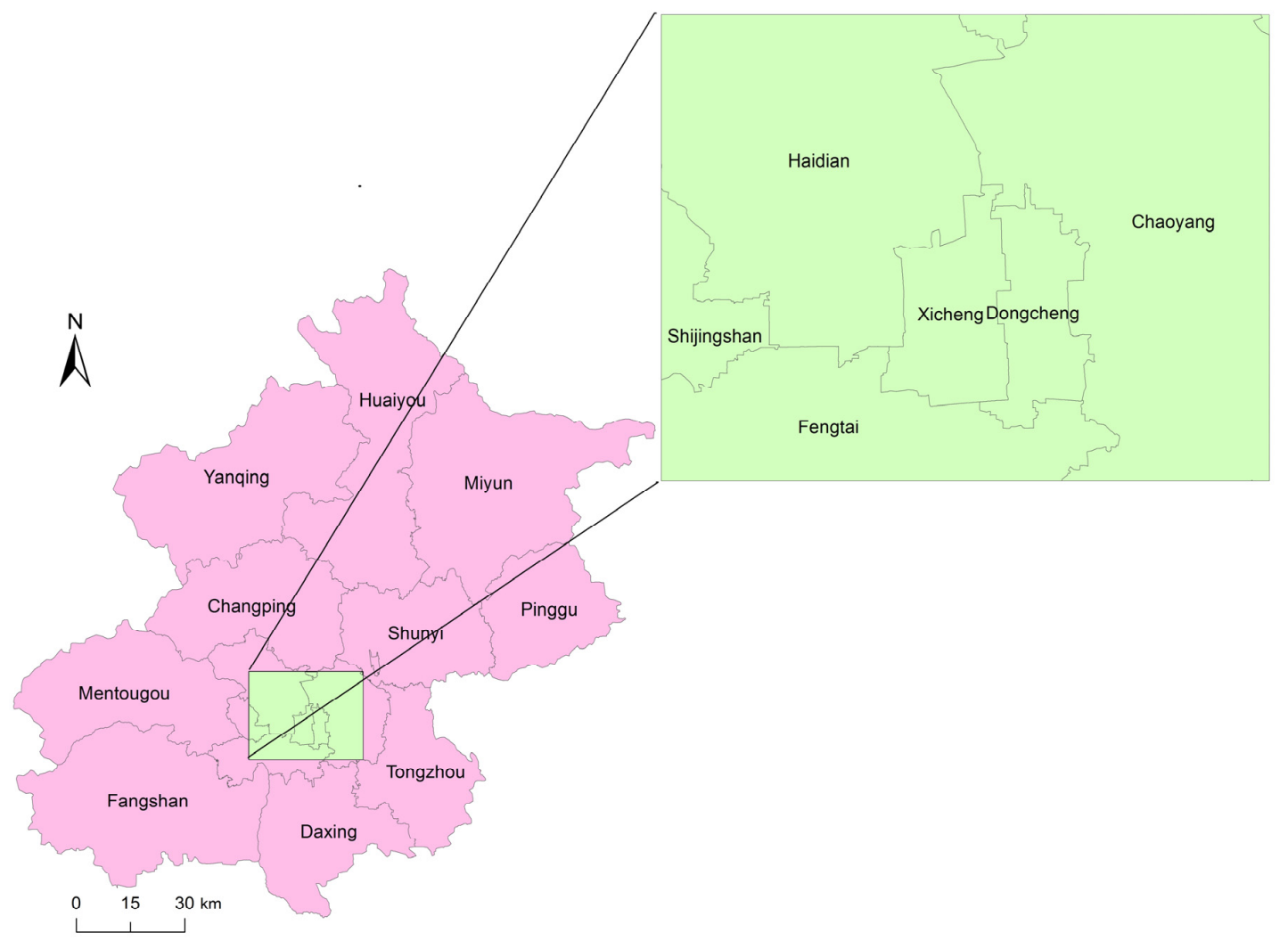

Figure 1. Study area in Beijing, denoted by a green rectangle.

We selected hospitals from the 2013 Beijing basic medical insurance-designated medical institutions, which are reviewed by social security departments and must sign an agreement with the health insurance agencies to provide medical services to insured people. The dataset contains information such as hospital names, grades, and addresses, from which we determined hospital locations. Moreover, we used the trips extracted from taxi trajectories to delineate the medical service areas and spatially influenced regions. The study area contains 78 hospitals including specialty hospitals and general hospitals, and their spatial distribution is shown in Figure 2.

\subsection{Calculating Pick-Up and Drop-Off Points}

To identify the DOPs and PUPs around the hospitals, we created multiple ring buffers at specified distances $20 \mathrm{~m}$ around hospitals and calculated the percentage of DOPs and PUPs in each ring, as shown in Figure 3. The percentage of DOPs and PUPs first increases and then decreases, finally reaching a peak in the buffer area of 200-220 m. Additionally, a 200-m threshold was used because it is an acceptable walking distance, and an empirical study found that most distances between taxi PUPs (or DOPs) and the actual origin points (or destination points) are less than $200 \mathrm{~m}$ [18]. Hence, we draw a 200-m buffer zone for each hospital, and there was no interaction between the buffers. In each buffer zone, trips with DOPs or PUPs falling inside the area were found. We assume that trips within these areas are made by patients seeking hospital services. In sum, we establish an OD (origin-destination) 
linkage using the above-mentioned vector from $\left(x_{0}, y_{0}, t_{0}\right)$ to $\left(x_{d}, y_{d}, t_{d}\right)$ for each patient in the GPS dataset. Next, we determined the weight of each grid according to the number of points that fall within the cell. For each hospital, the weight of a cell is the number of trips between a location inside the cell and the hospital. Finally, we constructed a $78 \times 720$ matrix. In this matrix, each hospital corresponds to a line, and the row vector formed by the line represents the spatial distribution characteristics of its patients, which can be used for the following clustering analysis.

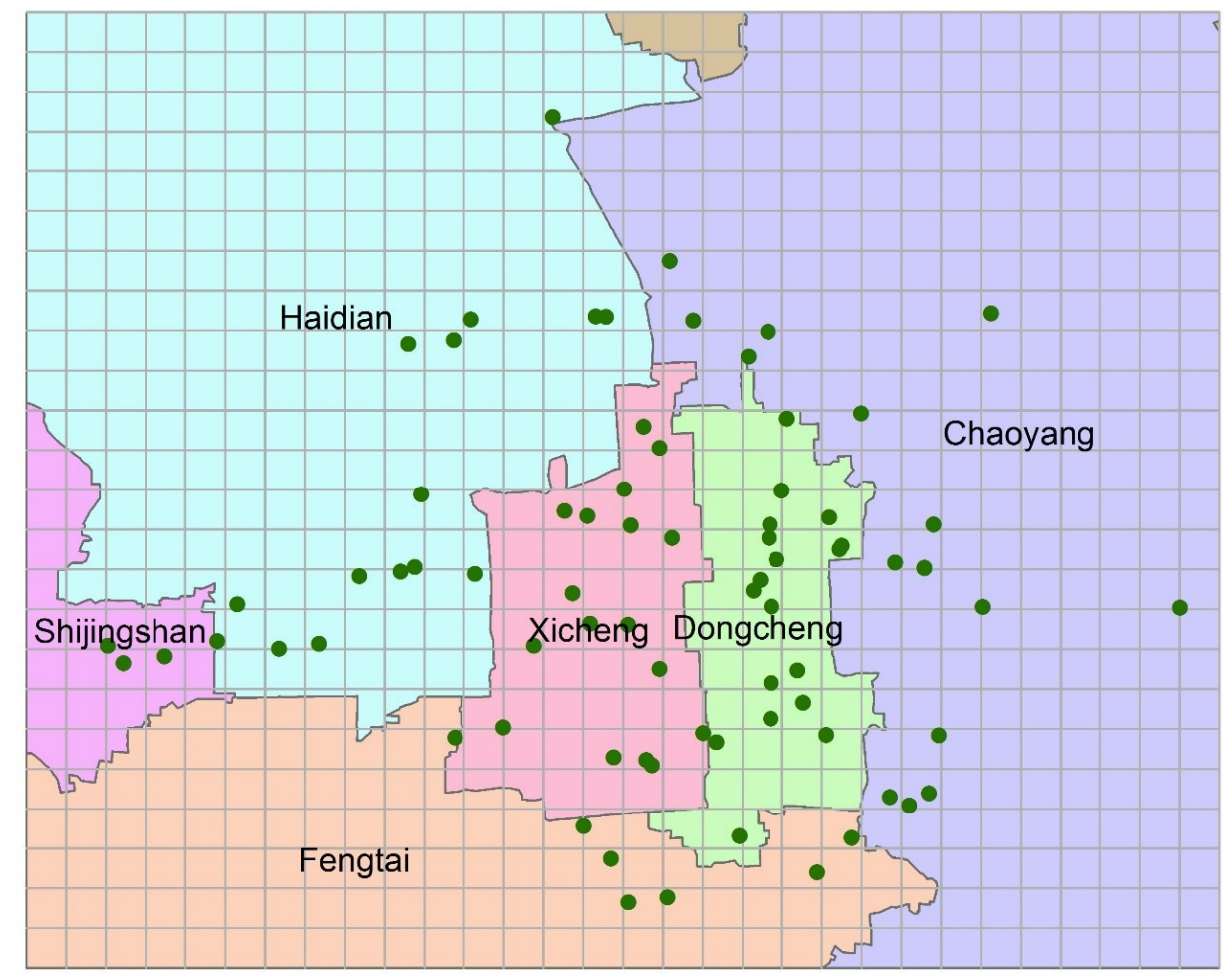

Figure 2. Spatial distribution of hospitals in the study area.

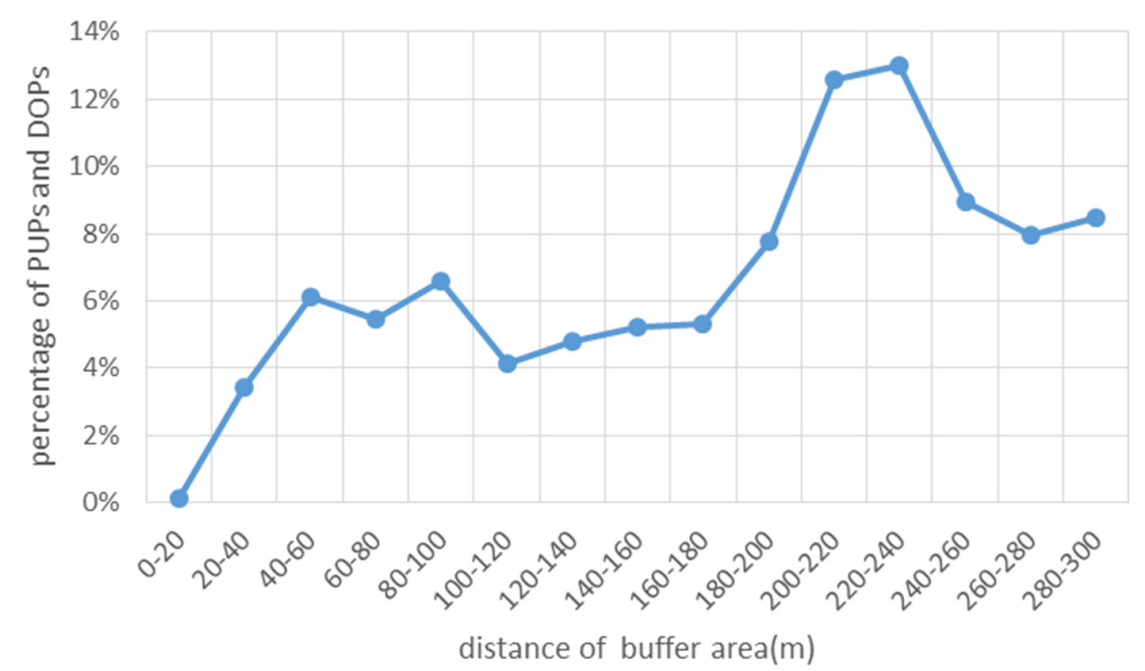

Figure 3. Percentage of PUPs and DOPs in buffer areas with different distances.

\section{Hospital Service Area and Patient Spatial Distribution Pattern}

The service area, an important spatial feature reflecting the attractiveness of a hospital, is related to the type of the hospital, distribution of patients, and existence of competitors. Patients' spatial 
choices are dependent on the distance between them [19]. An ideal way to assess patients' spatial choice behavior is to measure patient-to-hospital flows, which can be obtained from survey data. Because patient-to-hospital data are often unavailable, many scholars utilize the Huff model to estimate patient spatial distributions and hospital service areas [8,20]. Even when survey data are available, the sample size is generally small because of various limitations, such as the low efficiency of surveying, the strong requirement for time, human resources and finance. In addition, the quality of the survey data also has some limitations, such as low spatial precision, large time granularity, and weak data representation. Big geo-data have some advantages, such as large sample size and strong representation, and contain much spatial interaction; compared with survey data, big geo-data are more precise and objective in characterizing urban public facility characteristics [21].

In this study, we derived service areas based on massive patient-to-hospital data, which were obtained from taxi GPS data. To directly demonstrate hospital service areas, we rasterized and visualized the spatial distribution of all 78 hospitals' patients (Figure 4). We notice that most patients come from four districts (Dongcheng, Xicheng, Chaoyang, and Haidian) within the fifth beltway. The spatial distribution of patients is assumed to be similar to that of the overall population distribution. One reason for this distribution is that these districts belong to the core urban areas of Beijing, and the population density in these districts is the highest. Another reason may be that high-level hospitals are densely distributed in these areas, which attract a large number of people seeking medical services.

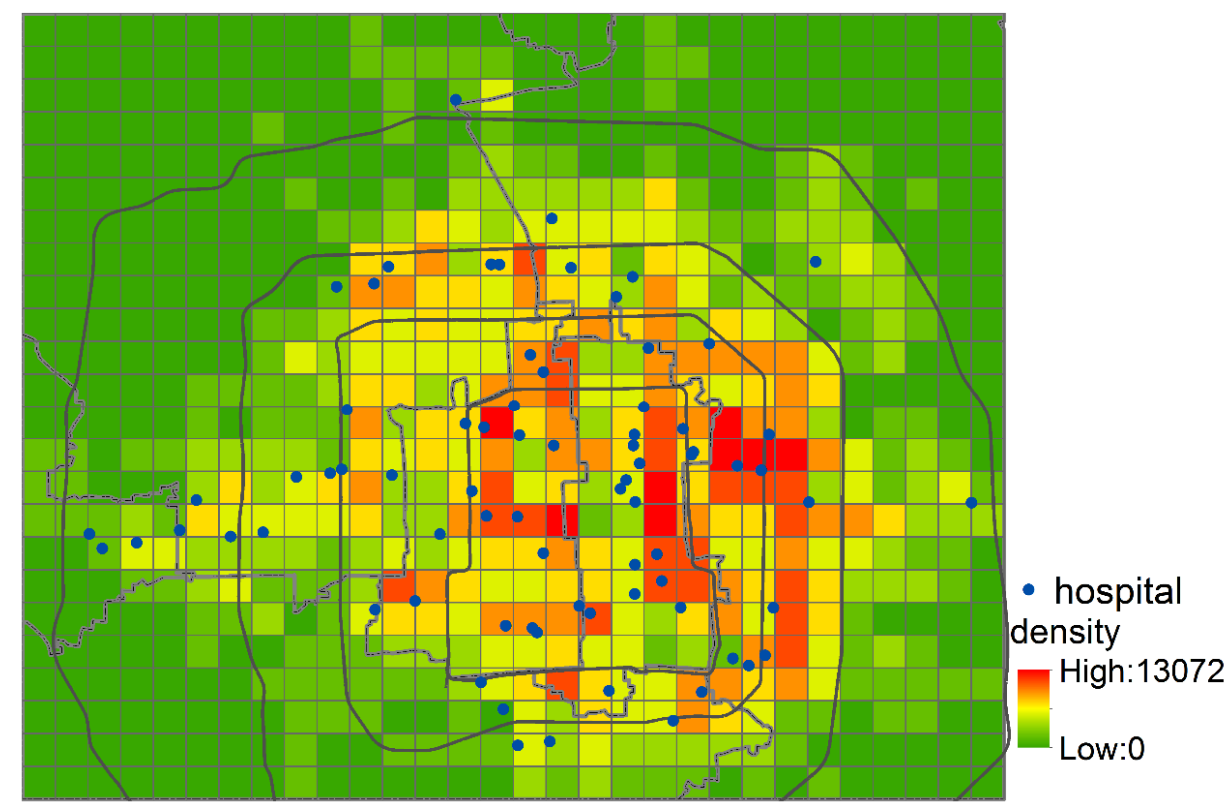

Figure 4. The overall hospital service areas.

To examine the hospital service areas in detail, we selected six general and specialty hospitals from the 78 hospitals as examples-(1) The First Affiliated Hospital of PLA General Hospital (\#304 Hospital); (2) Air Force General Hospital; (3) Peking University Third Hospital; (4) Peking University People's Hospital; (5) Xicheng Woman and Children Health Hospital; and (6) Fengtai Maternal \& Child Health Hospital (Figure 5) - and compared their service areas' characteristics. For a better understanding of the service areas of these hospitals, we first simply introduce these hospitals. The six hospitals can be categorized into three groups. First, the Air Force General Hospital and \#304 Hospital are both military hospitals. However, they not only serve the army but also provide medical services to general patients from all parts of the country. Second, Peking University Third Hospital and Peking University People's Hospital are general hospitals providing comprehensive medical care with scientific research and teaching components. Xicheng Woman and Children Health Hospital and Fengtai Maternal \& Child Health Hospital are specialty hospitals serving mainly women and children. 
As shown in Figure 5, the six hospitals' service areas vary substantially across the region, with patients mainly coming from the downtown area inside the fifth beltway. Although \#304 Hospital and Air Force General Hospital are both military hospitals and are located close to each other, the Euclidean distance between them is approximately $750 \mathrm{~m}$. The Air Force General Hospital has a larger service area and attracts more patients, which may be attributed to its specialty: dermatology. As for the second group of hospitals, the service area of People's Hospital covers mainly the Xicheng and Haidian districts, which are located between the north second beltway and the north third beltway. The service area of the Peking University Third Hospital is concentrated in the Haidian and adjacent regions. Compared with the Peking University Third Hospital, Peking University People's Hospital serves more patients and a larger area. Xicheng Woman and Children Health and Fengtai Maternal \& Child Health Hospital are local specialty hospitals, and their patients are mainly from the vicinity of the hospitals, with relatively fewer patients than the four other hospitals. Through the comparative analysis, we discover that most patients are concentrated in the areas surrounding the hospitals, indicating that people tend to seek medical services/care nearby. Some patients, however, would rather make a relatively long trip to visit a higher-level hospital.

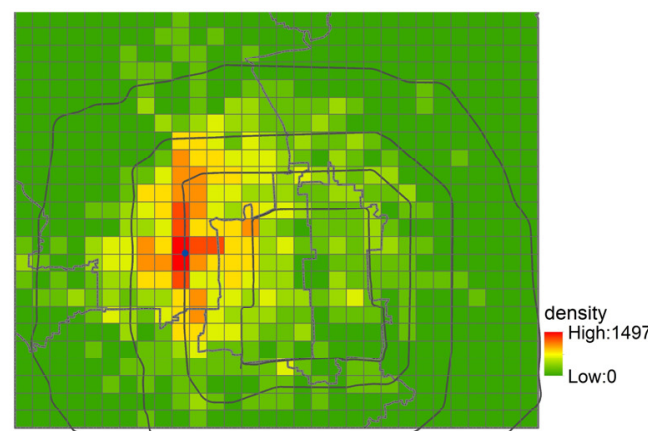

(a)

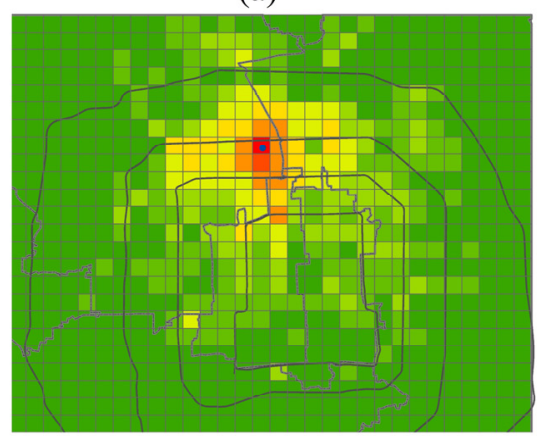

(c)

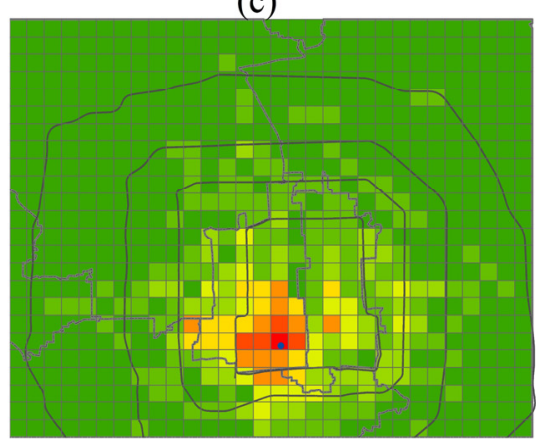

(e)

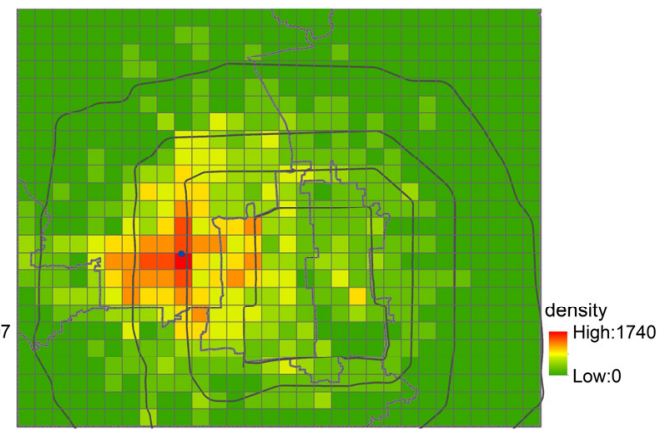

(b)

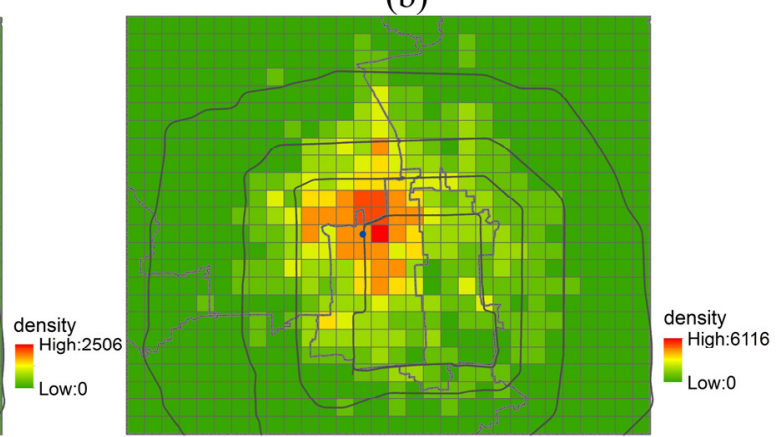

(d)

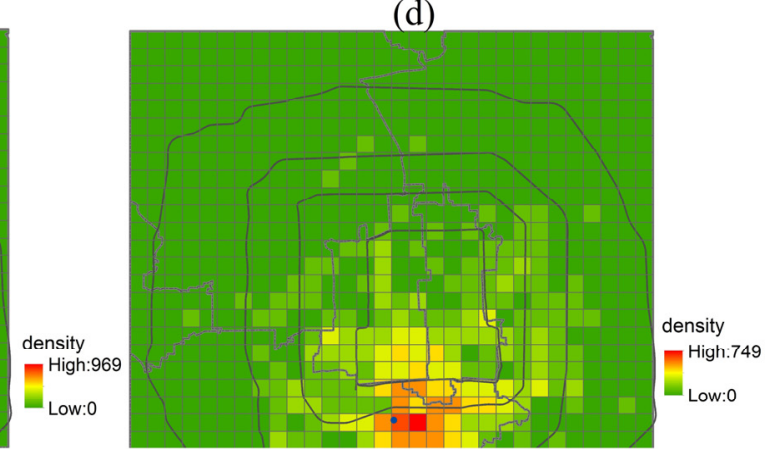

(f)

Figure 5. Hospital service areas. (a) The First Affiliated Hospital of PLA General Hospital (\#304 Hospital); (b) Air Force General Hospital; (c) Peking University Third Hospital; (d) Peking University People's Hospital; (e) Xicheng Woman and Children Health Hospital; (f) Fengtai Maternal \& Child Health Hospital. 
In the above sections, we visually examined the spatial distribution of patients based on the spatial interaction. Given that distance is widely regarded as an important factor of spatial interactions [22,23], we can quantify the spatial interaction patterns from the perspective of distance decay. In general, spatial interaction declines with an increase in distance, as also known as the distance decay effect. A distance decay function can be used to characterize the role of distance. The commonly used distance decay function includes an exponential function $\left(f(d)=\mathrm{C} e^{-\alpha d}, \alpha>0\right)$, a power function $\left(f(d)=C d^{-\beta}, \beta>0\right)$, and a Gaussian function $\left(f(d)=C d^{-\alpha d^{2}}, \alpha>0\right)$.

To examine the impact of the travel distance on hospital visits, we plotted the interaction intensity and distance in Figure 6 . The $x$-axis represents the distance in kilometers, and the $y$-axis represents the patient proportion $(p(d))$ under a certain distance. As shown in Figure $6, p(d)$ increases at first and reaches its peak when $d$ is approximately $2 \mathrm{~km}$, as the majority of movements occur in the central urban area. After that, the patient spatial distribution demonstrates an obvious distance decay trend: the number of patients decreases with distance. The probability distribution fits well with an exponential distance decay function $\left(R^{2}=0.91\right)$ and is consistent with existing findings observed from taxi data [24] and mobile phone data [25], and accords with the general law of people's service utilization.

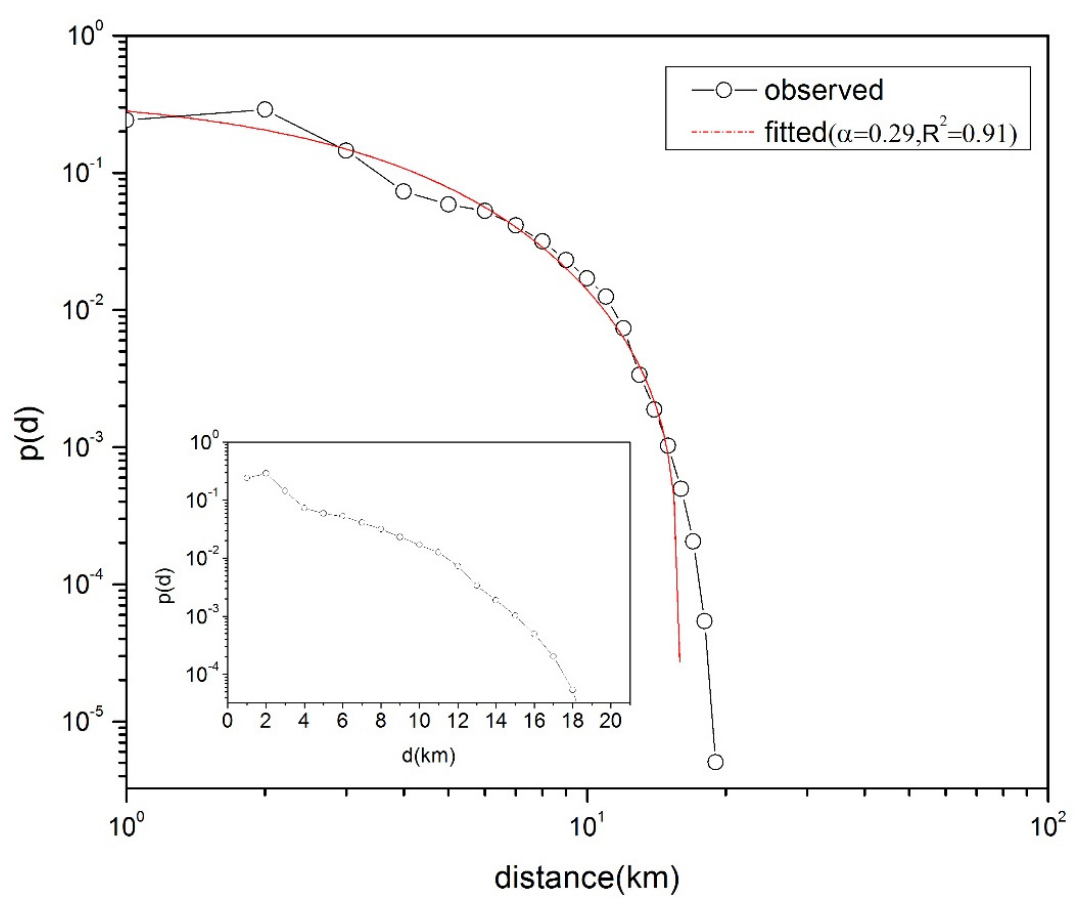

Figure 6. Profile of the distance decay function of all hospital visits.

Figure 6 depicts the overall distance decay of visits to all hospitals. However, different hospitals may exhibit different distance decay effects given their locations, specialty focus and other factors. Hence, we plotted the patient distributions for all the six hospitals to characterize the effect of distance (Figure 7). As shown in the figure, the patient distributions of the 6 hospitals are all affected by the distance. However, the attenuation velocities of the distance decay are not the same based on the varying decay rates $(\alpha)$ of the exponential distance decay functions. Here, we chose the decay rate $(\alpha)$ as the comparative factor because it can be used to quantify the role of distance in the spatial interaction between different hospitals and patients, and it can be used as an important parameter in the accessibility calculation model of medical facilities, with an important application value [26]. The distance decay rates of the same type of hospitals tend to be similar. Class-A military hospitals, Air Force General Hospital and \#304 Hospital have similar distribution patterns with similar distance decay rates: 0.107 and 0.13, respectively. The Peking University Third Hospital and the People's Hospital are university hospitals and have a similar distribution pattern. Compared with Peking 
University Third Hospital, People's Hospital has a smaller distance decay effect. Xicheng Woman and Children Health and Fengtai Maternal \& Child Health Hospital are local specialty hospitals. The average of $\alpha$ of the two hospitals is larger than the previous general hospitals, indicating that patients of these hospitals are more sensitive to travel distance. In general, residents usually take some factors into consideration when they choose hospitals for medical service. The first factor is the level or the type of the hospital. Class-A and general hospitals are much better than local hospitals. The second is distance [27]. Because money and time have costs, hospitals that are very far from home may not be a good choice. The spatial interaction between patients and hospitals we obtained is consistent with the actual situation.

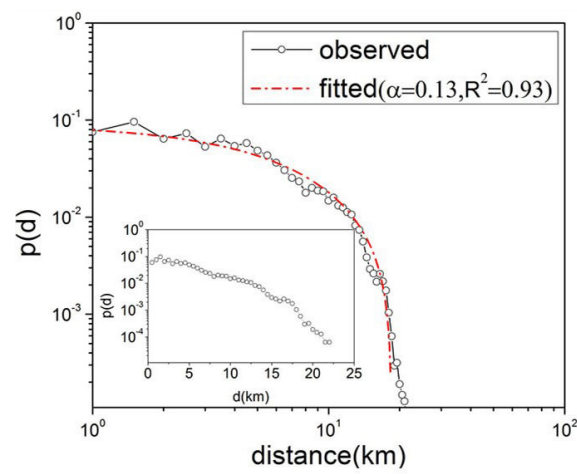

(a)

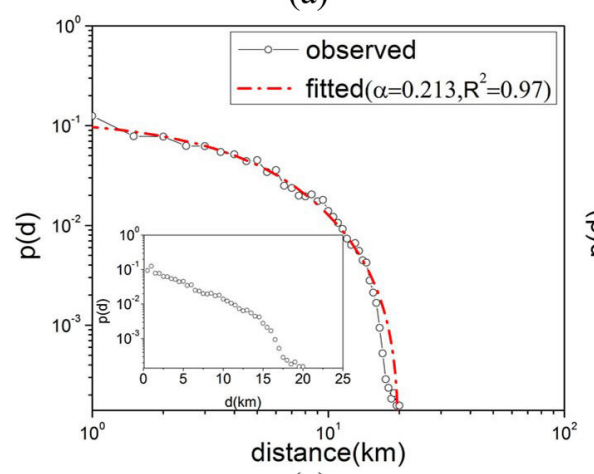

(c)

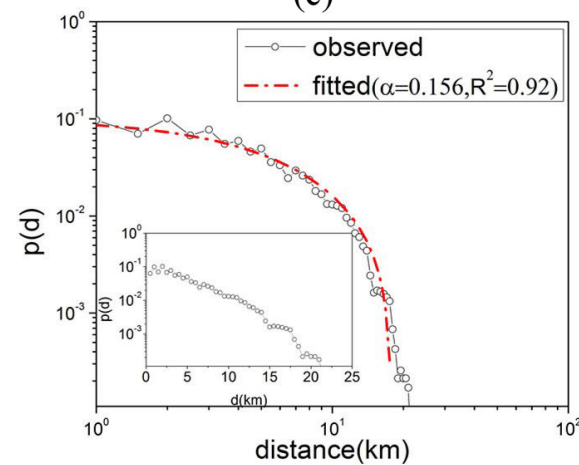

(e)

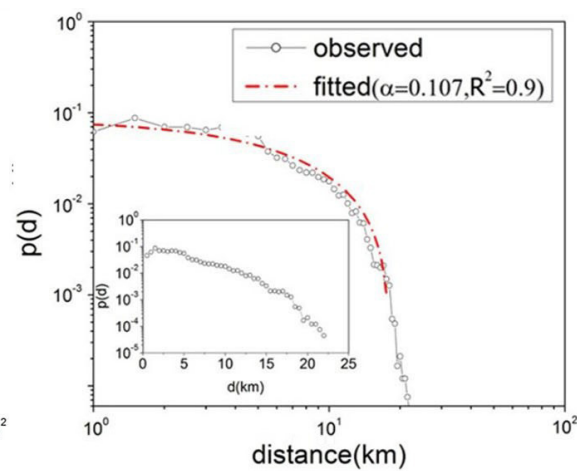

(b)

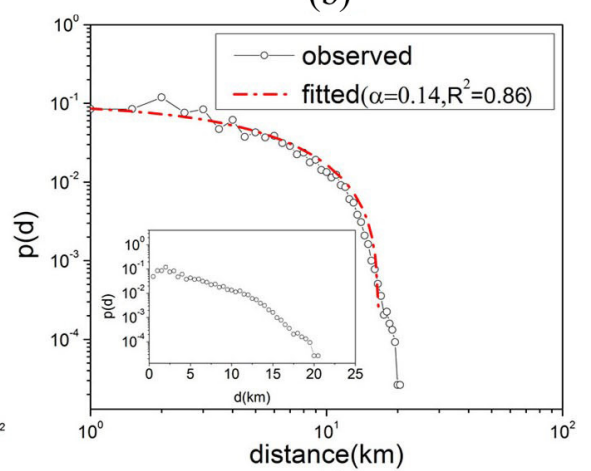

(d)

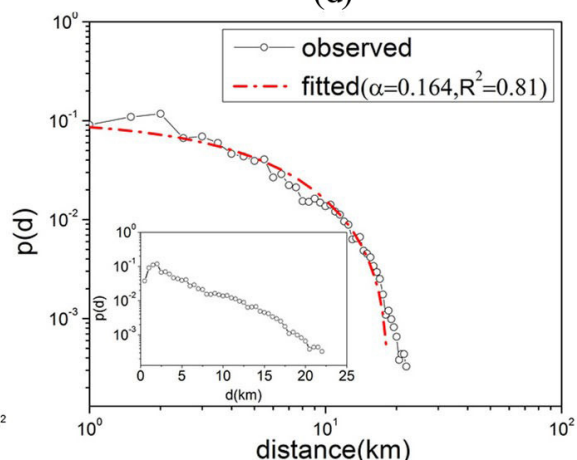

(f)

Figure 7. Distance distributions of typical hospitals' visits. (a) The First Affiliated Hospital of PLA General Hospital (\#304 Hospital); (b) Air Force General Hospital; (c) Peking University Third Hospital; (d) Peking University People's Hospital; (e) Xicheng Woman and Children Health; (f) Fengtai Maternal \& Child Health Hospital.

\section{Classifying Hospitals from a Spatial Interaction Perspective}

We have observed that different hospitals have different service areas with varying patient travel distances. Therefore, we try to classify hospitals based on the source of the patients. The traditional 
hospital classification is based on a number of criteria that were proposed based on hospital classification management standards. According to the nature of the business, hospitals can be divided into general hospitals and specialty hospitals. Depending on their specialty, hospitals can be divided into ENT (ears, nose, and throat) hospitals and cancer hospitals, among others. Based on the nature of the industry, hospitals can be divided into teaching hospitals, corporate hospitals, and military hospitals. In accordance with their service objectives, hospitals can also be divided into geriatric hospitals, pediatric hospitals, and women and children's hospitals, among others. Compared with the traditional classification of hospitals, our hospital classification is based on the spatial interaction strength between patients and hospitals and focuses on human mobility. This method can be used to classify hospitals because the spatial interaction between hospitals and patients can reflect hospital characteristics and can be easily obtained from taxi data.

\subsection{Methodology and Results}

In this section, we used a k-means clustering method, a segmentation clustering method based on partitioning, to cluster hospitals based on patient distribution characteristics, which were obtained from Section 2.3. The main advantages of this clustering algorithm are that the algorithm is fast and easy and suitable for large-scale data mining. Therefore, we chose the k-means clustering method to cluster the hospitals into $k$ categories. The specific process of the algorithm is as follows:

1. Assuming that all the observations are divided into k classes;

2. Selecting $k$ initial cluster centers;

3. Allocating observations to the nearest centers identified;

4. Computing the new cluster centers based on the allocated observations in 3 and repeating 2-3 times until the criterion function achieves convergence.

Generally, a criterion function is given by a squared error

$$
J_{C}=\sum_{i=1}^{k} \sum_{x \in C_{i}}\left|x-p_{i}\right|^{2}
$$

where $x$ represents observations, and $p_{i}$ is the average of the observations in cluster $c_{i}$.

In the clustering process, we used the logarithm of spatial interaction strength in each cell to reduce the variation in the overall interaction values observed, resulting in a better classification result. By comparing the classification results under different $k$ values, we found that, when the number of hospital types was 5 , the criterion function reached its minimum with a more reasonable classification result.

The classification result is shown in Figure 8. We see that most specialty hospitals were grouped into one class, military hospitals into another class, and university hospitals into a third class. The specific classification results are shown in Table 1.

Table 1. Classification results of hospitals.

\begin{tabular}{|c|c|c|}
\hline Cluster & Hospital Description & Example Hospitals \\
\hline 1 & Specialty hospitals as the main type & $\begin{array}{l}\text { Xicheng Woman and Children Health Hospital, Cancer Hospital } \\
\text { Chinese Academy of Sciences, etc. }\end{array}$ \\
\hline 2 & Military hospitals as the main type & $\begin{array}{l}\text { Air Force General Hospital, The First Affiliated Hospital of PLA } \\
\text { General Hospital (304), } 466 \text { Hospital, The Second Artillery Force } \\
\text { of the Chinese People's Liberation Army General Hospital, etc. }\end{array}$ \\
\hline 3 & University hospitals as the main type & $\begin{array}{l}\text { Peking University People's Hospital, Peking University Third } \\
\text { Hospital, Beijing Anding Hospital, etc. }\end{array}$ \\
\hline 4 & General hospitals as the main type & Beijing No. 6 Hospital, Beijing Chaoyang Hospital, etc. \\
\hline 5 & Mixed type & $\begin{array}{l}\text { Chinese PLA General Hospital, Beijing Cancer Hospital, Beijing } \\
\text { hospital of Chinese Traditional and Western Medicine, etc. }\end{array}$ \\
\hline
\end{tabular}




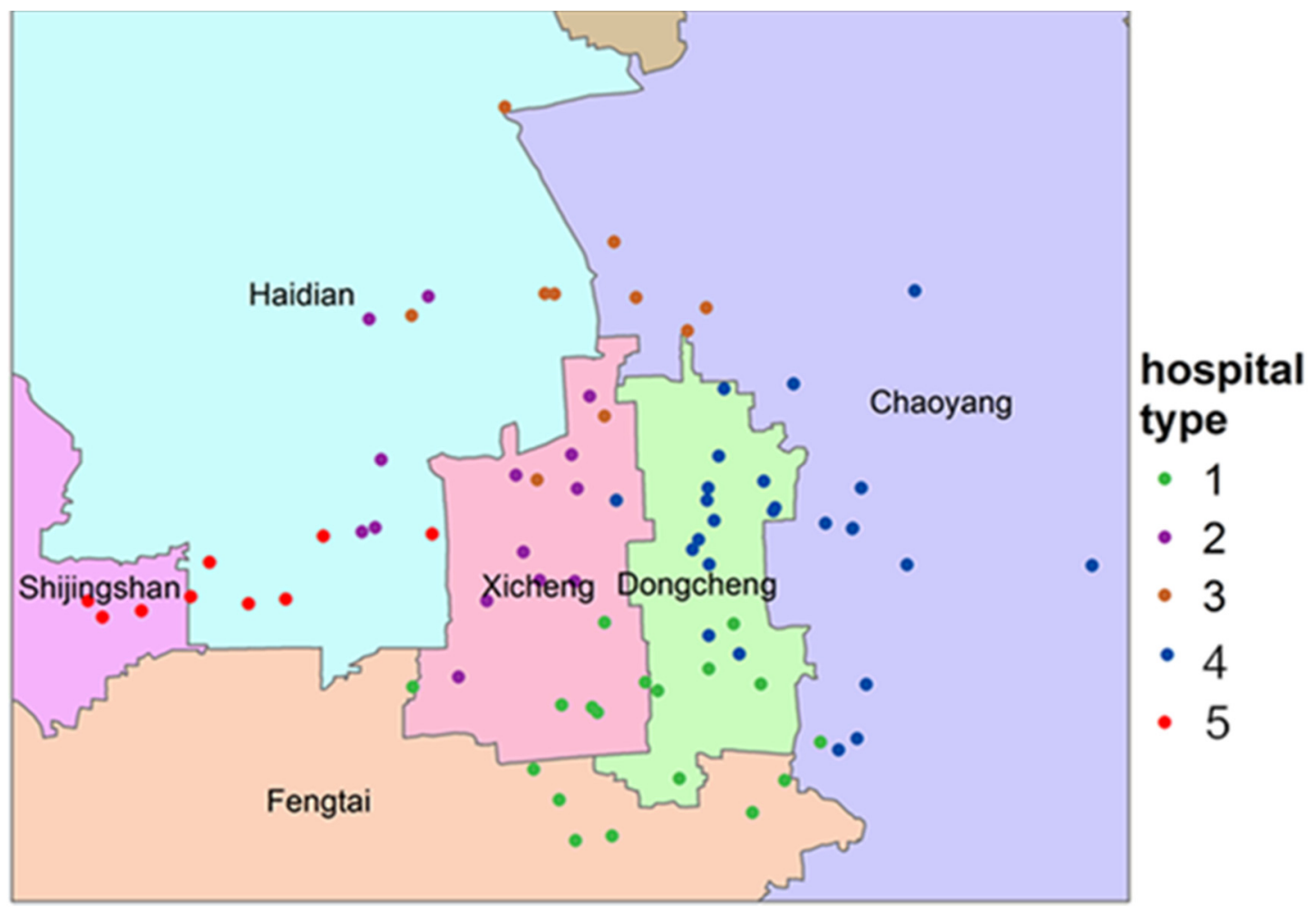

Figure 8. Classification result of 78 hospitals.

\subsection{Analyzing the Patient Distribution Characteristics for Each Type of Hospital}

We plotted the probability distribution of each type of hospital (Figure 9). As seen in the figure, the patient distribution for each type of hospital also shows a distance decay trend, but the attenuation rates vary. Similarly, we used exponential functions to fit these distributions and calculated the attenuation index to examine the relationship between the patient distribution of each type of hospital and distance. Combining patient distribution and the distance decay rate $\alpha$, we analyzed all types of hospitals. The first class is specialty hospitals, and the distribution shows significant distance attenuation with an exponent of 0.33 . The second class is Class-A military hospitals with high authority, attracting a large number of people every day with a smaller attenuation rate $(\alpha=0.28)$. The third class is university hospitals, such as military hospitals, and patient visits decrease slowly with distance. The fourth class is general hospitals: compared with specialty hospitals, the curve's exponent a is larger, indicating that patient visits are more affected by distance. This class includes many small municipal general hospitals that serve people nearby, such as Beijing No. 6 Hospital. The last class mixes all types of hospitals, and its attenuation index is very similar to that obtained from all hospitals. 


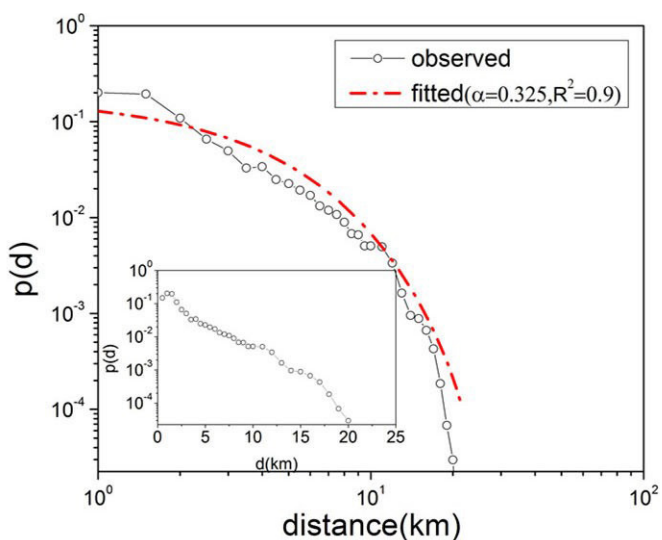

(a)

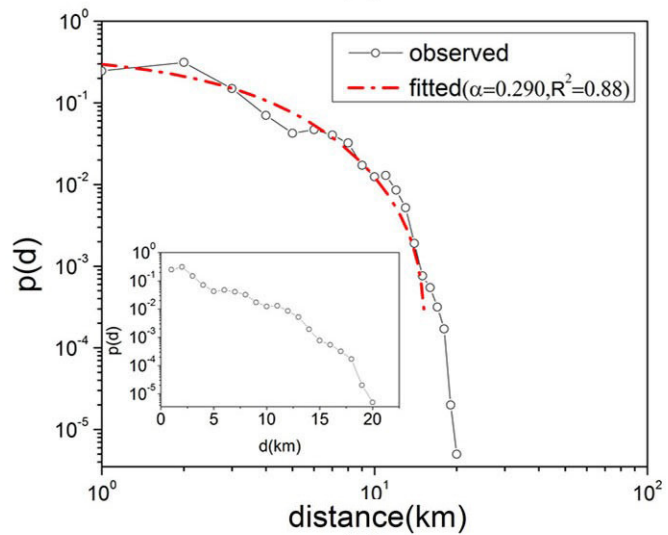

(c)

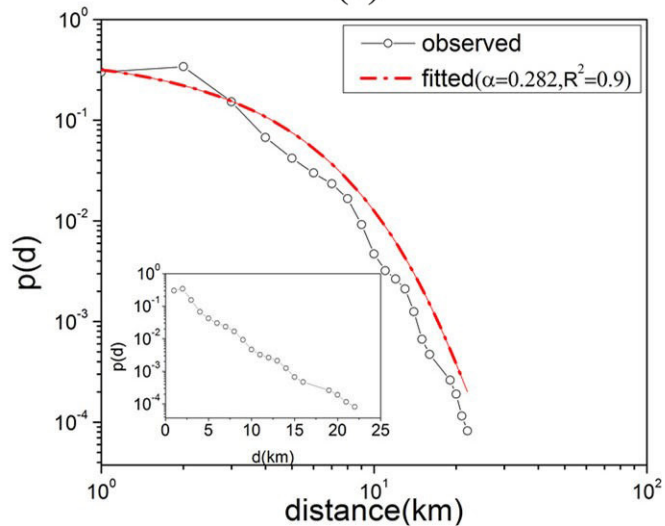

(e)

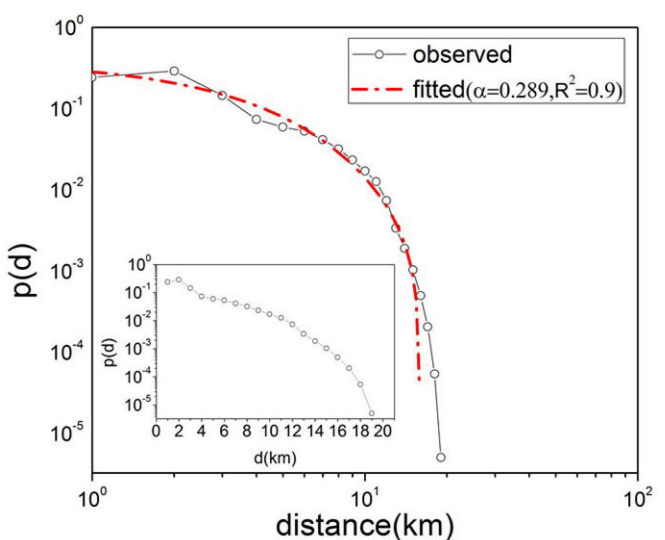

(b)

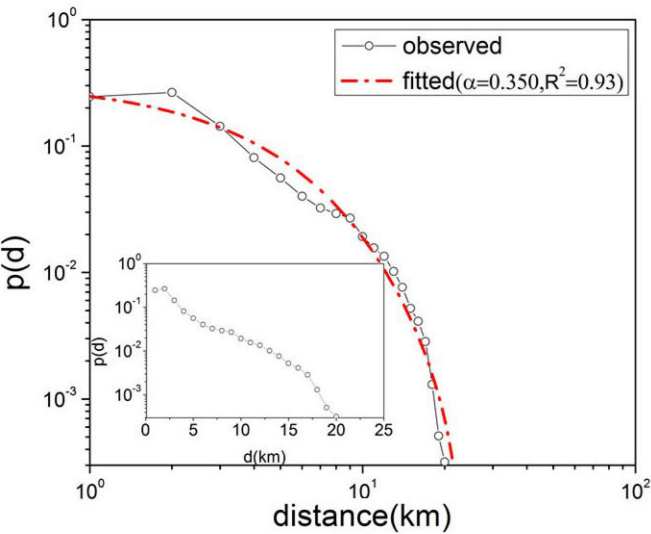

(d)

Figure 9. Distance distribution associated with each class of hospital. (a-e) represent the clusters of hospitals separately.

\section{Discussion and Conclusions}

Public facility characteristics have been of great interest to city and regional planners. Existing studies investigating public facility characteristics are mainly based on static data. This study aimed at investigating public facility characteristics from the perspective of spatial interaction. Using the pick-ups and drop-offs extracted from a large taxi dataset, we applied an unsupervised classification method to study 78 hospitals located in six districts in Beijing. We classified hospitals in the study area into 5 types and investigated the hospitals' patient distribution characteristics and service areas. Our findings include two aspects, as follows. First, the main medical service areas of all the hospitals are concentrated in the Haidian, Chaoyang, Dongcheng and Xicheng districts, where the 
population and hospitals are dense. Meanwhile, the patient distribution of all hospitals demonstrates the distance decay effect, following an exponential distribution. Second, the patient distribution is related to the type of hospital. Among them, the patient distribution of high-level university hospitals and military hospitals is less affected by distance attenuation, but specialty hospitals and municipal general hospitals are more sensitive to distance. In conclusion, travel distance plays an important role in choosing a hospital for medical help, but when people suffer from a serious illness such as cancer, they tend to choose hospitals of high quality and are less sensitive to the travel distance.

Compared with static data, the taxi data we used in this study contain large individual tracking data, which allowed us to study patient mobility patterns and spatial interaction with hospitals. The classification method we utilized in our study investigated the similarity between hospitals from a spatial interaction perspective, reflecting a bottom-up analytical perspective. The characteristics of a hospital can be better understood by considering its interaction with patients. That is to say, the classification of hospitals in our study relies mainly on human activities, which can reflect the concept of social sensing, revealing the spatial-temporal distribution, link, and process of socio-economic phenomena from human spatial behavior characteristics extracted through spatial big geo-data [12]. Our study provides important insights for policy makers to make adjustments to the current spatial configuration of hospitals in order to provide accessible medical services while helping reduce traffic pressure caused by hospital visits. Moreover, distance decay exponents in patient-hospital interactions can be applied to study health care access.

There are some limitations to our study. Taxi data can cover only a portion of all patients because some patients may go to hospitals by car or public transport, while others who live very close to hospitals are likely to go on foot. As a result, this paper examined only the characteristics of taxi travel, and the research findings may not be generalizable to the entire population. Furthermore, we did not analyze the PUPs and DOPs separately and did not consider the impact of population density on patient spatial distribution. Additionally, using the patient distribution to determine the surrounding traffic conditions might be insufficient. In the future, we will include more data, such as public transportation card records, to provide a better understanding of the spatial distribution of patients. We will also analyze PUPs and DOPs separately and consider population density in our future research. Finally, we will combine patient distribution characteristics and road traffic information to assess areas in which congestion is mainly caused by hospital visits and to provide constructive suggestions for urban planners to develop possible effective remedies.

Acknowledgments: This work was supported by National Natural Science Foundation of China (Nos. 41428102 and 41625003).

Author Contributions: Yu Liu, Jing Zhang, and Daoqin Tong conceived and designed the experiments; Xiaoqing Kong performed the experiments; Xiaoqing Kong and Yuxia Wang analyzed the data; Xiaoqing Kong, Jing Zhang and Yu Liu wrote the paper.

Conflicts of Interest: The authors declare no conflict of interest.

\section{References}

1. Luo, W.; Wang, F. Measures of spatial accessibility to health care in a GIS environment: Synthesis and a case study in the Chicago region. Environ. Plan. B Plan. Des. 2003, 30, 865-884. [CrossRef]

2. Wang, F.; Wei, L. Assessing spatial and nonspatial factors for healthcare access: Towards an integrated approach to defining health professional shortage areas. Health Place 2005, 11, 131-146. [CrossRef] [PubMed]

3. Tsou, K.W.; Hung, Y.T.; Chang, Y.L. An accessibility-based integrated measure of relative spatial equity in urban public facilities. Cities 2005, 22, 424-435. [CrossRef]

4. Song, Z.; Chen, W.; Zhang, G.; Zhang, L. Spatial accessibility to public service facilities and its measurement approaches. Prog. Geogr. 2010, 29, 1217-1224.

5. Gittelsohn, A.; Powe, N.R. Small area variations in health care delivery in Maryland. Health Serv. Res. 1995, 30, 295-317. [PubMed] 
6. Guagliardo, M.F.; Jablonski, K.A.; Joseph, J.G.; Goodman, D.C. Do pediatric hospitalizations have a unique geography? BMC Health Serv. Res. 2004, 4, 137-140. [CrossRef] [PubMed]

7. Klauss, G.; Staub, L.; Widmer, M.; Busato, A. Hospital service areas-A new tool for health care planning in Switzerland. BMC Health Serv. Res. 2005, 5, 1-15. [CrossRef] [PubMed]

8. Jia, P.; Xierali, I.M.; Wang, F. Evaluating and re-demarcating the Hospital Service Areas in Florida. Appl. Geogr. 2014, 60, 248-253. [CrossRef]

9. Talen, E.; Anselin, L. Assessing spatial equity: An evaluation of measures of accessibility to public playgrounds. Environ. Plan. A 1998, 30, 595-613. [CrossRef]

10. Chang, H.S.; Liao, C.H. Exploring an integrated method for measuring the relative spatial equity in public facilities in the context of urban parks. Cities 2011, 28, 361-371. [CrossRef]

11. Taleai, M.; Sliuzas, R.; Flacke, J. An integrated framework to evaluate the equity of urban public facilities using spatial multi-criteria analysis. Cities 2014, 40, 56-69. [CrossRef]

12. Liu, Y.; Liu, X.; Gao, S.; Gong, L.; Kang, C.; Zhi, Y.; Chi, G.; Shi, L. Social sensing: A new approach to understanding our socioeconomic environments. Ann. Assoc. Am. Geogr. 2015, 105, 512-530. [CrossRef]

13. Liu, Y.; Sui, Z.; Kang, C.; Gao, Y. Uncovering patterns of inter-urban trip and spatial interaction from social media check-in data. PLoS ONE 2014, 9, e86026. [CrossRef] [PubMed]

14. Ratti, C.; Sobolevsky, S.; Calabrese, F.; Reades, J.; Martino, M.; Claxton, R.; Strogatz, S.H. Redrawing the map of Great Britain from a network of human interactions. PLoS ONE 2010, 5, e14248. [CrossRef] [PubMed]

15. Roth, C.; Kang, S.M.; Batty, M.; Barthélemy, M. Structure of urban movements: Polycentric activity and entangled hierarchical flows. PLoS ONE 2011, 6, e15923. [CrossRef] [PubMed]

16. Liu, X.; Kang, C.; Gong, L.; Liu, Y. Incorporating spatial interaction patterns in classifying and understanding urban land use. Int. J. Geogr. Inf. Sci. 2016, 30, 334-350. [CrossRef]

17. Beijing Municipal Commission of Development and Reform. Available online: http://www.bjpc.gov.cn/ zmhd/hdft/201603/t9986424.htm (accessed on 9 September 2016).

18. Gong, L.; Liu, X.; Wu, L.; Liu, Y. Inferring trip purposes and uncovering travel patterns from taxi trajectory data. Cartogr. Geogr. Inf. Sci. 2016, 43, 103-114. [CrossRef]

19. Ritsema van Eck, J.R.; de Jong, T. Accessibility analysis and spatial competition effects in the context of GIS-supported service location planning. Comput. Environ. Urban Syst. 1999, 23, 75-89. [CrossRef]

20. Wang, Y.F.; Zhang, C. GIS and gravity polygon based service area analysis of public facility: Case study of hospitals in Pudong new area. Econ. Geogr. 2005, 25, 800-803.

21. Wu, Z.F.; Chai, Y.W.; Dang, A.R.; Gong, J.H.; Gao, S.; Yue, Y.; Li, D.; Liu, L.; Liu, X.J.; Liu, Y.; et al. Geography interact with big data: Dialogue and reflection. Geogr. Res. 2015, 34, 2207-2221.

22. Tobler, W.R. A computer movie simulating urban growth in the Detroit region. Econ. Geogr. 1970, 46, $234-240$. [CrossRef]

23. Miller, H.J. Tobler's First law and spatial analysis. Ann. Assoc. Am. Geogr. 2004, 94, 284-289. [CrossRef]

24. Liang, X.; Zheng, X.; Lv, W.; Zhu, T.; Xu, K. The scaling of human mobility by taxis is exponential. Phys. A 2012, 391, 2135-2144. [CrossRef]

25. Kang, C.; Ma, X.; Tong, D.; Liu, Y. Intra-urban human mobility patterns: An urban morphology perspective. Phys. A 2012, 391, 1702-1717. [CrossRef]

26. Wang, F. Measurement, optimization, and impact of health care accessibility: A methodological review. Ann. Assoc. Am. Geogr. 2012, 102, 1104-1112. [CrossRef] [PubMed]

27. Zhu, Z.; Cao, Z.; Zeng, D.D. An analysis of Beijing HFMD patients mobility pattern during seeking treatment. In Proceedings of the International Conference on Smart Health, Beijing, China, 3-4 August 2013.

(C) 2017 by the authors; licensee MDPI, Basel, Switzerland. This article is an open access article distributed under the terms and conditions of the Creative Commons Attribution (CC BY) license (http:/ / creativecommons.org/licenses/by/4.0/). 